\title{
Diagnostic value of DACT-2 methylation in serum of prostate cancer patients
}

\author{
Ting Lan ${ }^{1,2 \#}$, Lingyu Yin ${ }^{2 \#}$, Haoliang Zhang ${ }^{2,3 \#}$, Bing Gu${ }^{1,2,3}$, Ping $\mathrm{Ma}^{1,2,3}$, Shibao $\mathrm{Li}^{1,2,3}$, Hongchun $\mathrm{Li}^{2,3}$ \\ ${ }^{1}$ Xuzhou Key Laboratory of Laboratory Diagnostics, Xuzhou Medical University, Xuzhou, China; ${ }^{2}$ School of Medical Technology, Xuzhou Medical \\ University, Xuzhou, China; ${ }^{3}$ Department of Laboratory Medicine, Affiliated Hospital of Xuzhou Medical University, Xuzhou, China \\ Contributions: (I) Conception and design: S Li, H Li; (II) Administrative support: S Li, P Ma; (III) Provision of study materials or patients: H Zhang, \\ L Yin; (IV) Collection and assembly of data: T Lan, L Yin; (V) Data analysis and interpretation: T Lan, L Yin; (VI) Manuscript writing: All authors; \\ (VII) Final approval of manuscript: All authors. \\ "These authors contributed equally to this work. \\ Correspondence to: Shibao Li, PhD, MD. Department of Laboratory Medicine, Affiliated Hospital of Xuzhou Medical University, 99 West Huaihai \\ Road, Xuzhou 221002, China. Email: sdjnshlb@xzhmu.edu.cn; Hongchun Li, MD. Department of Laboratory Medicine, Affiliated Hospital of \\ Xuzhou Medical University, Xuzhou 221002, China. Email: 13775891123@163.com.
}

\begin{abstract}
Background Currently, prostate cancer (PCa) remains a hard nut to crack for the medical community. Therefore, the identification and development of novel biomarkers that can accurately diagnose disease and predict prognosis are of paramount importance. The objective of this study was to examine the clinical value of DACT-2 promoter methylation in serum of patients with $\mathrm{PCa}$, to discover a potential diagnostic marker for PCa.
\end{abstract}

Methods: We investigated the methylation status of DACT-2 in the serum of 64 patients with PCa, 22 patients with benign prostatic hyperplasia (BPH), and 47 healthy subjects by methylation-specific PCR (MSP) and real-time methylation-specific PCR (QMSP). Further, we evaluated the relationship between DACT-2 methylation and clinic pathological parameters. Receiver operating characteristic (ROC) curve analysis was applied to assess the sensitivity, specificity, and diagnostic value of DACT-2 methylation and PSA levels.

Results: The results of MSP and QMSP showed that the level of methylation of DACT-2 promoter in patients with PCa was significantly higher than that in patients with BPH and healthy subjects. The PCa patients Gleason score and tumor node metastasis (TNM) positively correlated with promoter methylation level of serum DACT-2. The DACT-2 methylation rate was 0.745 with a sensitivity of $81.8 \%$, and a specificity of $75.0 \%$, the sensitivity, and specificity of PSA was $80.1 \%$ and $59.4 \%$. ROC curve results displayed that the diagnostic value of DACT-2 is superior to PSA.

Conclusions: Our study confirms that the level of methylation of the DACT-2 promoter in patients with $\mathrm{PCa}$ is much higher than that in patients with benign prostatic hyperplasia (BPH) and healthy subjects, suggesting that DACT-2 methylation in serum is a potential biomarker of PCa.

Keywords: DNA methylation; DACT-2; prostate cancer

Submitted Jul 28, 2020. Accepted for publication Nov 17, 2020.

doi: 10.21037/apm-20-1496

View this article at: http://dx.doi.org/10.21037/apm-20-1496

\section{Introduction}

Prostate cancer (PCa) is one of the most emergent health issues globally, accounting for the second cause of cancer deaths in males $(1,2)$. Recently, the incidence of PCa is increasing in numerous countries at pace with the steadily ceaseless development of society, especially the rapid expansion of the economy $(3,4)$. Moreover, there has been evidence that the lifestyle and living environment of the public are two potential risk factors for PCa (5). Currently, 
PCa screening remains to rely on serum prostate-specific antigen (PSA) testing and random biopsy $(6,7)$. However, the biopsy is an invasive test, which is not an appropriate screening. Meanwhile, PSA has low specificity, with falsepositive results in patients with benign prostatic hyperplasia $(\mathrm{BPH})$ and biopsy is only positive in around $25 \%$ of patients with PSA in the range between 2 and $10 \mu \mathrm{g} / \mathrm{L}$. PSA screening and random biopsy strategies may induce that they diagnosed the other diseases and missing the PCa. The US Preventive Health Task Force issued a "D" grade for PSA-based screening in 2012 because they both over-diagnose low-risk disease and under diagnose highrisk cancers (8). Therefore, it is demanding to explore new biomarkers for the diagnosis and prognosis of $\mathrm{PCa}$.

Epigenetic changes play a vital role in the development and progression of different cancers, including PCa. In particular, hypermethylation of $\mathrm{CpG}$ islands of the gene promoter region leads to reduced expression of gene (9). The original study has demonstrated that circulating tumor DNA (ctDNA) has the potential function for prediction, molecular analysis, and monitoring. Thus, ctDNA in the blood of tumor patients can be used as biomarkers (10). So far, circulating tum75 or DNA methylation as a biomarker of cancer has been used in the early detection of numerous cancers (11). Several studies have confirmed that abnormal methylations of serum EphA5, PITX3, PD-L1, DEFB1, PITX2, CRMP4 genes are involved in the malignant evolution of $\mathrm{PCa}$, which can be used as a biomarker for assessing the risk of PCa (12-17). Accordingly, hypermethylation of the gene promoter may be a potential biomarker for the diagnosis of PCa.

The human DACT gene family contains three members, namely dact- 1 , dact- 2 , and dact- 3 , with 2,1 , and $5 \mathrm{CpG}$ islands in the promoter region, respectively. Therefore, the presence of aberrant methylation of the DACT promoter may lead to a lower expression of mRNA, which in turn affects its normal biological function, including its role in the development of the tumor $(18,19)$. Recent studies have shown that DACT-2 is down-regulated by its promoter methylation in various tumors, including colon cancer, gastric cancer, thyroid cancer, and esophageal cancer (20-23). It is hypothesized that DACT-2 may be a potential tumor suppressor gene involved in tumor progression. Our previous studies have also confirmed that DACT-2 is downregulated by methylation in both prostate cancer cells and prostate cancer tissues (24). However, it is unclear whether the presence of DACT-2 gene methylation is present in the serum of patients with PCa. In this study we investigated the methylation status of DACT-2 promoter in serum of patients with PCa. We analyzed the relationship between the serum DACT-2 methylation and clinic pathological parameters in PCa for the better of the development of a novel and potential biomarker during diagnosis in PCa.

We present the following article in accordance with the STARD reporting checklist (available at http://dx.doi. org/10.21037/apm-20-1496).

\section{Methods}

\section{Eligibility criteria}

Between January 2015 and December 2016, 135 participants, including 66 patients with $\mathrm{PCa}, 22$ patients with benign prostatic hyperplasia and 47 healthy control, were consecutively enrolled in this prospective observational study conducted in the Affiliated Hospital of Xuzhou Medical University. The study was conducted in accordance with the Declaration of Helsinki (as revised in 2013) and was approved by the Ethics Committee of the Affiliated Hospital of Xuzhou Medical University (XYFY2019KL113-01). Written informed consent was obtained from all participants.

Inclusion criteria of $\mathrm{PCa}$ were as follows: (I) male that was pathologically diagnosed with PCa were included in the PCa group; (II) male that was diagnosed with benign prostatic hyperplasia (BPH) were used color Doppler ultrasound examinations as benign group; (III) men with normal findings both at physical and color Doppler ultrasound examinations were included in the healthy group.

Exclusion criteria of PCa were as follows: (I) history of other primary or secondary tumors; (II) history of other comorbidities (e.g., hypertension, diabetes, heart disease, renal disease, etc.); (III) pre-operative chemotherapy or radiotherapy; (IV) hormonal treatment before surgery.

After color Doppler ultrasound examinations, blood samples were collected from patients with $\mathrm{BPH}$ and healthy controls, blood samples of patients with PCa were obtained daily before treatment, including radical prostatectomy, pre-operative chemotherapy, and radiotherapy. The pathological parameters, Gleason scores were taken from biopsies or prostatectomies. All samples were separated from serum and rapidly frozen at $-80{ }^{\circ} \mathrm{C}$. According to the 7th edition of the AJCC staging system, PCa patients were evaluated for the pathological stage. Clinical and biological data from the patients with $\mathrm{PCa}$ and $\mathrm{BPH}$ are listed in Table 1 . 
Table 1 Patient clinical and histological characteristic

\begin{tabular}{lccc}
\hline & $\begin{array}{c}\text { Prostate } \\
\text { cancer (\%) }\end{array}$ & BPH (\%) & $\begin{array}{c}\text { Health } \\
\text { Persons (\%) }\end{array}$ \\
\hline Case, $\mathrm{n}$ & 64 & 22 & 47 \\
Age (mean \pm SD, years) & $71.1 \pm 6.7$ & $70.8 \pm 7.3$ & $69.5 \pm 10.3$ \\
PSA (ng/mL) & & & \\
$<4$ & $4(6.3)$ & $21(95.5)$ & $46(97.9)$ \\
$4-10$ & $15(23.4)$ & $0(0)$ & $1(2.1)$ \\
$>10$ & $45(70.3)$ & $1(4.5)$ & \\
TNM & & & \\
T1 & $9(14.1)$ & & \\
T2 & $12(18.8)$ & & \\
T3 & $13(20.3)$ & & \\
T4 & $30(46.8)$ & \\
Gleason score & & \\
6 -7 & $18(28.1)$ & \\
$8-10$ & $46(71.9)$ & \\
\hline
\end{tabular}

$\mathrm{BPH}$, benign prostatic hyperplasia; PSA, prostate specific antigen; TNM, tumor node metastasis.

\section{Serum DNA extraction and sodium bisulfite modification}

According to the manufacturer's instructions, $200 \mu \mathrm{L}$ of serum was used to extract DNA from each sample with the aid of Serum/Plasma Circulating DNA Kit (TIANGEN, Beijing, China). Then, the eluted $100 \mu \mathrm{L}$ of serum DNA was stored at $-20{ }^{\circ} \mathrm{C}$ for subsequent experiments. The extracted DNA was modified based on the operating manual for the EZ DNA Methylation-Gold Kit (ZYMO Research Co, Orange, CA) kit, and the modified DNA was stored at $-20^{\circ} \mathrm{C}$.

\section{Methylation-specific polymerase chain reaction (MSP)}

The MSP primer sequences applied to amplify the modified DNA in this study were derived from the previous research (20). The sequences of MSP primers are as follows: 5'-GCGCGTGTAGATTTCGTTTTTCGC-3' (MF) and 5'-AACCCCACGAACGACGCCG-3' (MR); 5'-TTGGGGTGTGTG TAGATT TTG TTT TTTGT-3' (UF) and 5'-CCCAAACCCCACAAA CAA CAC CA-3' (UR). The MSP amplification system used in this experiment was $20 \mu \mathrm{L}$, consisting of $10 \mu \mathrm{L}$ of Taq PCR MasterMix, $10 \mu \mathrm{L}$ of nuclease-free water, $1 \mu \mathrm{L}$ of each upstream and downstream primers and $1 \mu \mathrm{L}$ of modified DNA. The amplification conditions involved in this reaction are as follows: $95^{\circ} \mathrm{C} 5 \mathrm{~min},\left(95^{\circ} \mathrm{C} 30 \mathrm{~s}, 58^{\circ} \mathrm{C} 30 \mathrm{~s}, 72{ }^{\circ} \mathrm{C} 30 \mathrm{~s}\right)$ $\times 38,72^{\circ} \mathrm{C} 7 \mathrm{~min}$. The size of methylated and unmethylated products was about 152 and $161 \mathrm{bp}$, respectively, separated on a $3 \%$ agarose gel and visualized under UV illumination.

\section{Real-time methylation-specific PCR}

As above, the DNA of the serums of 47 healthy subjects, 22 prostatic hyperplasia subjects and $64 \mathrm{PCa}$ subjects was extracted and subjected to bisulfite modification. The level of methylation of the DACT-2 promoter was examined on an ABI 7500 system (Applied Biosystems) using the MSP primers, and amplification conditions described above. The methylation level of the DACT-2 gene was calculated according to the following formula: M\%= $100 \%$ x [number of copies of methylated DNA/(number of copies of methylated + unmethylated DNA)]. In this study, the levels of DACT-2 methylation rate at 0.745 were defined as positive according to the ROC (receiver operating characteristic) curves. The levels of PSA $>7.56 \mathrm{ng} / \mathrm{mL}$ were defined as positive based on the reference range provided by the kit.

\section{Statistical analysis}

The experimental data were analyzed using SPSS 16.0 and GraphPad Prism 5.0 software. Differences between groups were compared by $t$-test. The differences between the three groups were assessed by the one-way ANOVA test and the relationship between DACT-2 methylation level and clinic pathological parameters was compared by the chi-square test. A receiver operating characteristic (ROC) curve was established to evaluate the diagnostic value of DACT-2 in PCa patients. When the P value is less than 0.05 , the difference is considered statistically significant.

\section{Results}

\section{The methylation status of DACT-2 in serum}

First of all, we detected the levels of methylated DACT2 in the serum of 64 patients with PCa, 22 patients with $\mathrm{BPH}$, and 47 healthy controls by methylation-specific PCR (MSP-PCR). Interestingly, methylated DACT-2 appeared in the serum of 34 (53.1\%) patients with PCa. In contrast, the frequency of the methylation of DACT-2 in serum was 


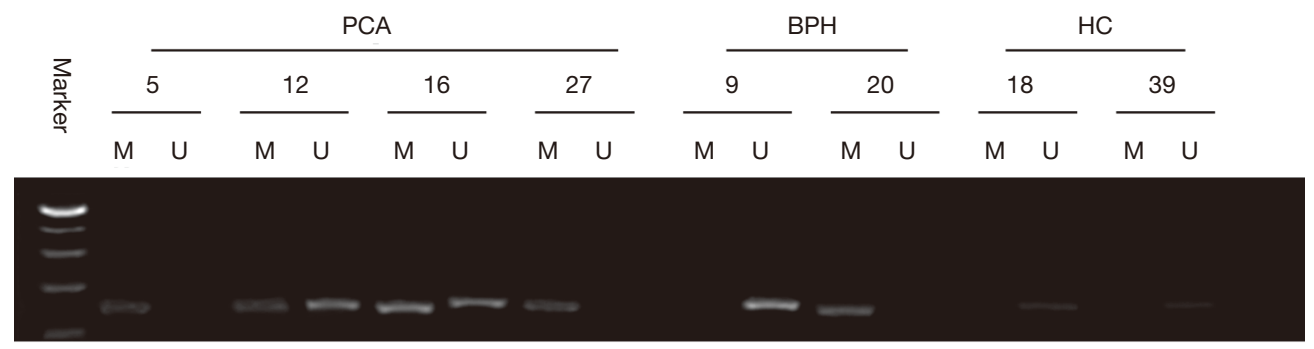

Figure 1 DACT-2 promoter methylation status in serum samples from prostate cancer (PCa) patients, benign prostatic hyperplasia (BPH) patients and healthy subjects controls (HCs) by methylation-specific PCR (MSP). M and U represent the amplification products of the methylated and unmethylated primers, respectively.

lower in BPH (9.1\%) and healthy subjects (4.3\%) (Figure 1).

\section{Relationship between the serum of DACT-2 methylation status and clinical pathological-parameters}

To elucidate the clinical significance of DACT-2 methylation in serum of patients with $\mathrm{PCa}$, we used Methylation-specific polymerase chain reaction (MSP) to analyze the association between DACT-2 methylation and clinic pathological parameters of PCa. The results showed a significant association between DACT-2 aberrant methylation and advanced pathological stage $(\mathrm{P}=0.027)$ and high Gleason score $(\mathrm{P}=0.011)$. However, there was no correlation between DACT-2 methylation and age $(\mathrm{P}>0.05)$ or preoperative PSA levels $(\mathrm{P}>0.05)$. The results of the statistical analysis are listed in Table 2.

\section{The rate of DACT-2 methylation in serum}

The rate of DACT-2 methylation in all serum samples was determined using real-time fluorescence MSP. We discovered that the methylation rate of the DACT-2 gene in serum DNA in patients with $\mathrm{PCa}$ was higher than that in $\mathrm{BPH}$ patients and healthy subjects (Figure $2 A$ ). To clarify the diagnostic value of PSA and serum DACT-2 promoter methylation rates in $\mathrm{PCa}$, the ROC curve was used to analysis the preoperative serum PSA level and DACT2 promoter methylation rate in serum. The ROC curve revealed a more favorable diagnostic performance of serum DACT-2 methylation rate than serum PSA concentrations in these samples. When the optimal cut-off point of DACT2 methylation rate was 0.745 with a sensitivity of $81.8 \%$ and a specificity of $75.0 \%$, the serum PSA value cut-off was $7.56(\mathrm{ng} / \mathrm{mL})$, with a sensitivity of $80.1 \%$ and a specificity of $59.4 \%$ (Figure 2B).

\section{Correlation between the methylation rate of DACT-2 and clinicopathologic features}

By analyzing histopathological parameters of DACT2 methylation rate, DACT-2 methylation rate was independent of median age $(\mathrm{P}>0.05)$ and PSA serum concentration $(\mathrm{P}>0.05)$. However, the methylation rate of the DACT-2 promoter in PCa patients is related to the Gleason score and the TNM stage. By the Realtime methylation-specific PCR our results exhibited that among PCa patients, the higher Gleason score [8-10] has a higher methylation rate of the DACT-2 promoter than the low Gleason score [6-7] (Figure 3A). Consistent with a higher rate of methylation of the serum DACT-2 promoter appeared in $\mathrm{PCa}$ with an advanced pathological stage (Figure 3B).

\section{Discussion}

Currently, the early diagnosis of PCa mainly relies on prostate-specific antigen PSA screening. Due to its low specificity for PCa, the detection of enhanced PSA levels not only results in the false positive diagnosis of $\mathrm{PCa}$ but also leads to a large number of unnecessary and invasive prostate biopsies $(6,25)$. Therefore, it is reasonably critical to identify diagnostic biomarkers with high sensitivity, specificity, and non-invasion for early diagnosis, improvement of prognosis, and survival rate of patients with $\mathrm{PCa}$.

Increasing evidence has shown that circulating tumor DNA (ctDNA) is a more appropriate and non-invasive liquid biopsy biomarker, which is easily detectable in a patient's plasma at an early stage of the disease (26-28). 
Table 2 Relationship between the serum of DACT-2 methylation status and clinical pathological-parameters

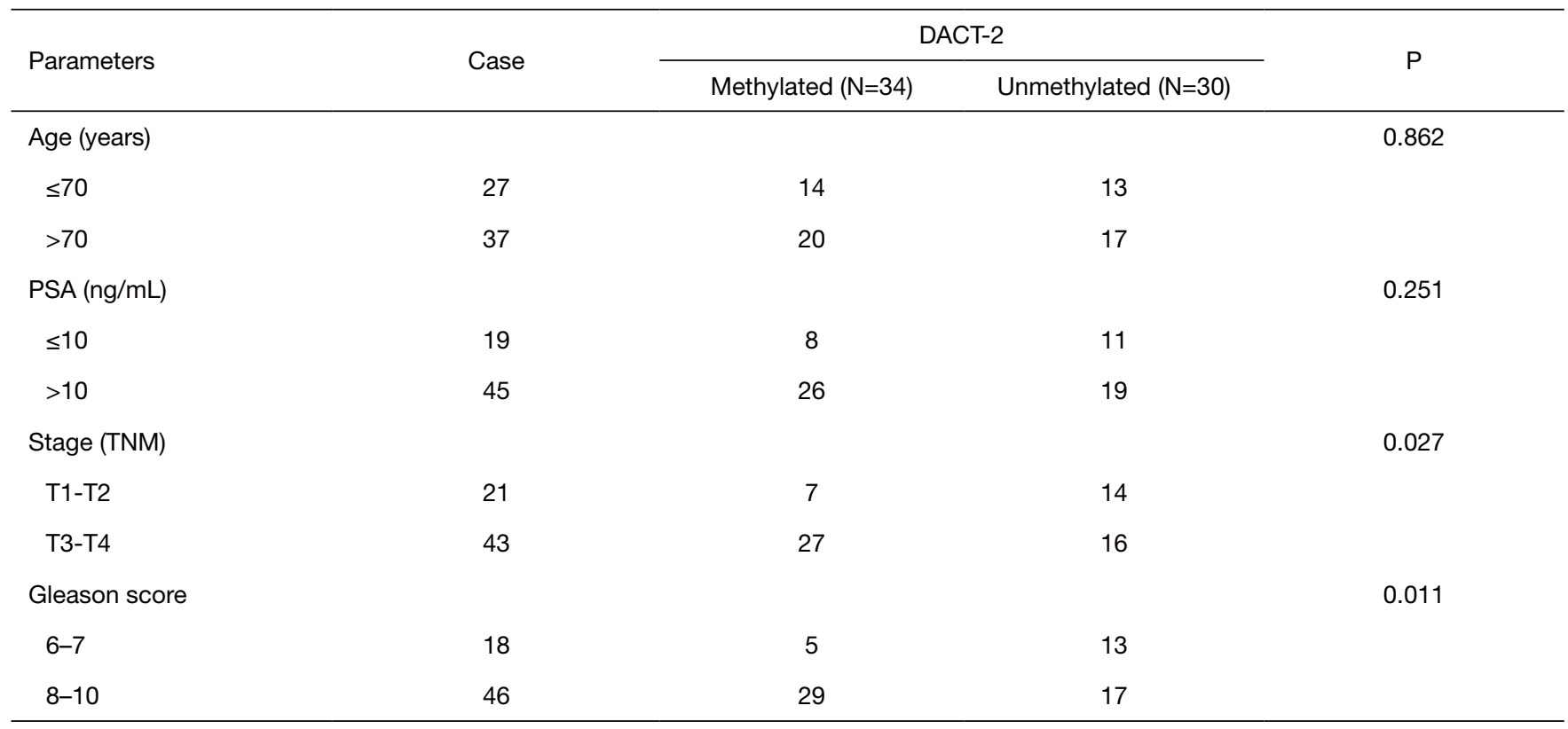

PSA, prostate specific antigen; TNM, tumor node metastasis.
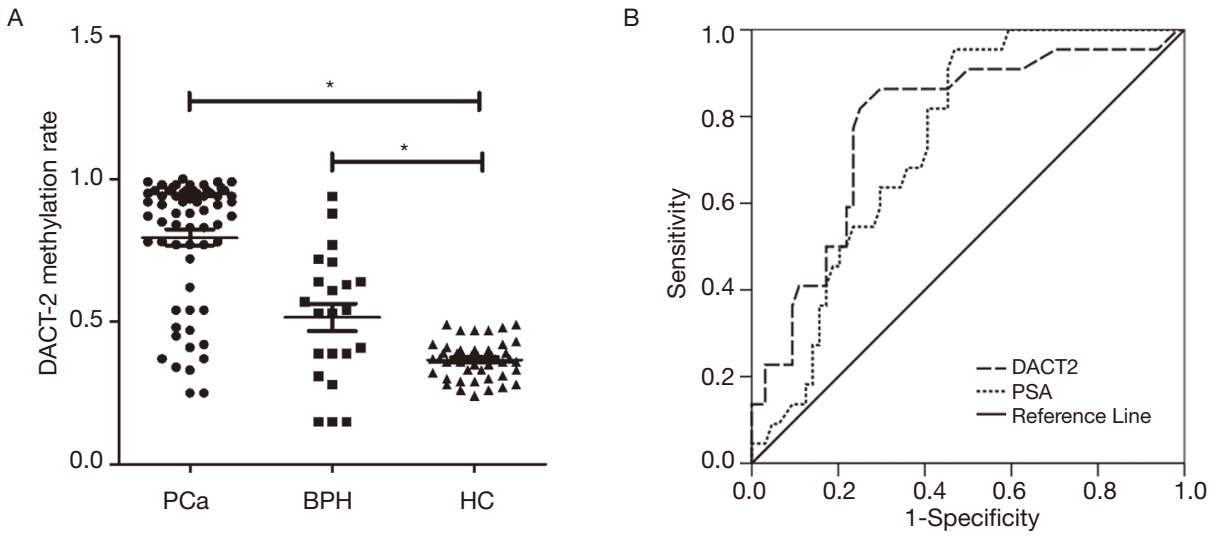

Figure 2 Methylation rate of DACT-2 in serum of prostate cancer (PCa) patients, benign prostatic hyperplasia (BPH) patients and healthy subjects controls (HCs). (A) The distribution of DACT-2 methylation rate in PCa patients, benign prostatic hyperplasia (BPH) patients and healthy subjects controls (HCs). (B) The receiver operating characteristic (ROC) curve of DACT-2 gene methylation rate (cutoff value of 0.745 ) and prostate specific antigen (PSA) level (cutoff value of $7.56 \mathrm{ng} / \mathrm{mL}$ ). ${ }^{*} \mathrm{P}<0.05$.

In particular, tumor-specific DNA methylation can be reflected in the ctDNA of cancer patients, demonstrating its potential for widespread clinical application in cancer detection (29,30). Given this general idea, we mainly probed into the methylated expression of DACT-2 in patients with PCa. Results of MSP showed that compared with $\mathrm{BPH}$ and healthy subjects, the methylated DACT-2 was highly present in the serum of patients with PCa. The above results are consistent observed in prostate cancer cells and prostate cancer tissues. Also, the discovery of this study is accordant with previous findings regarding the performance of DACT-2 in other tumors, suggesting that DACT-2 exhibits highly methylated in a variety of tumors (20-23). Guo et al. have validated that the hypermethylation 
A

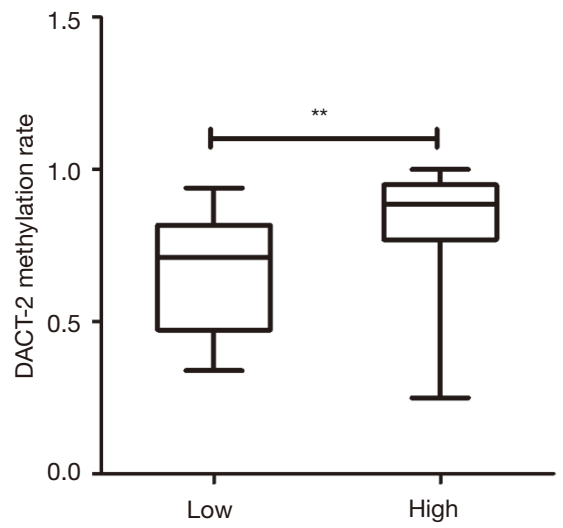

B

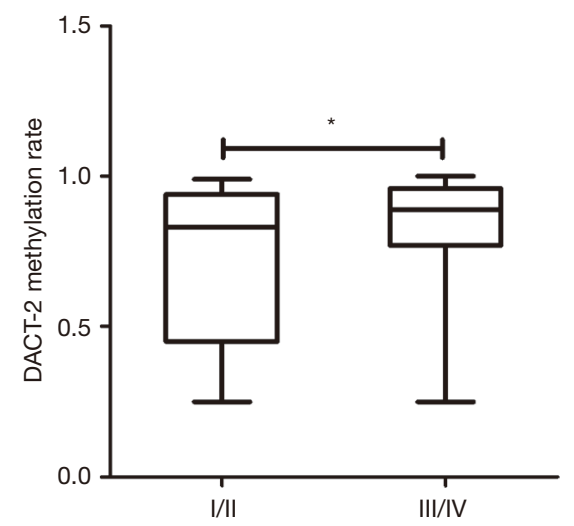

Figure 3 Clinicopathological features and DACT2 methylation rate in prostate cancer patients. (A) The Real-time methylation-specific PCR analysis correlation between DACT-2 methylation rate and Gleason Score, ${ }^{* *}$ represents a statistically significant difference of $\mathrm{P}<0.01$. (B) the Real-time methylation-specific PCR analysis correlation between DACT-2 methylation rate and tumor node metastasis (TNM) stage, *shows a statistically significant difference of $\mathrm{P}<0.05$.

of DACT-2 effectively promoted the progression of esophageal cancer (23). Moreover, several other studies have demonstrated that methylated DACT-2 can contribute to the development of tumors, including colon cancer, thyroid cancer, and breast cancer $(21,22,31)$. All the above findings validate that DNA methylation plays a crucial role in the carcinogenesis of tumors, so we further explored the clinical value of DACT-2 methylation in patients with PCa. Our analysis suggested that methylated DACT-2 was correlated with the Gleason score and TNM stage, and DACT-2 methylation expressed more frequently in high Gleason scores and patients with advanced PCa. Moreover, no correlation between the methylated DACT- 2 and median age $(\mathrm{P}=0.862)$, preoperative PSA levels $(\mathrm{P}=0.251)$ was observed in prostate carcinoma samples. In conclusion, the above results indicated that DACT-2 might act as a tumor suppressor for PCa.

To analyze the correlation between methylation of DACT-2 and clinic pathological parameters of PCa, we further explored the significance of the DACT-2 methylation rate in PCa by QMSP. We observed that the methylation rate of DACT-2 in the serum of patients with PCa was significantly higher than that in patients with $\mathrm{BPH}$ and healthy subjects. Moreover, we investigated the clinical significance of DACT-2 methylation rates in PCa, with a view to better understanding the diagnostic value of DACT-2 in PCa. Consistent with previous research, the experimental results displayed that the DACT-2 methylation rate of patients with high Gleason scores and advanced PCa was much higher than that of $\mathrm{BPH}$ and healthy subjects, which means the DACT-2 methylation plays an extraordinary role in the development and progression of PCa. In light of the fact that PCa screening is still dependent on PSA screening, we compared the diagnostic value of DACT-2 with PSA to detect PCa. ROC curve stated clearly that the sensitivity and specificity of DACT-2 were slightly higher than that of PSA, which proved that DACT-2 possessed a preferable diagnostic ability for PCa. Therefore, the above findings are sufficient to authenticate that DACT-2 methylation is a potential and clinically significant biomarker for the diagnosis of PCa.

Our findings demonstrate that methylation of DACT-2 is involved in the evolution of $\mathrm{PCa}$, which could potentially make DACT-2 a valuable biomarker for PCa diagnosis. The specific mechanism about how DACT-2 promotes the development of PCa needs to be further explored.

\section{Acknowledgments}

The authors want to recognize the cooperation of the patients, controls and investigators for their participation in this study, especially the staff of Medical Laboratory Section in Xuzhou Medical University Affiliated Hospital, China. Funding: The study was supported by the grant from 
the Jiang Su Provincial Medical Youth Talent (No. QNRC2016781) and the Natural Science Foundation of China (No.81702061).

\section{Footnote}

Reporting Checklist: The authors have completed the STARD reporting checklist. Available at http://dx.doi.org/10.21037/ apm-20-1496

Data Sharing Statement: Available at http://dx.doi. org/10.21037/apm-20-1496

Peer Review File: Available at http://dx.doi.org/10.21037/ apm-20-1496

Conflicts of Interest: All authors have completed the ICMJE uniform disclosure form (available at http://dx.doi. org/10.21037/apm-20-1496). The authors have no conflicts of interest to declare.

Ethical Statement: The authors are accountable for all aspects of the work in ensuring that questions related to the accuracy or integrity of any part of the work are appropriately investigated and resolved. The study was conducted in accordance with the Declaration of Helsinki (as revised in 2013) and was approved the Ethics Committee of the Affiliated Hospital of Xuzhou Medical University (XYFY2019-KL113-01). Written informed consent was taken from all the patients.

Open Access Statement: This is an Open Access article distributed in accordance with the Creative Commons Attribution-NonCommercial-NoDerivs 4.0 International License (CC BY-NC-ND 4.0), which permits the noncommercial replication and distribution of the article with the strict proviso that no changes or edits are made and the original work is properly cited (including links to both the formal publication through the relevant DOI and the license). See: https://creativecommons.org/licenses/by-nc-nd/4.0/.

\section{References}

1. Siegel RL, Miller KD, Jemal A. Cancer statistics, 2016. CA Cancer J Clin 2016;66:7-30.

2. Smith RA, Andrews K, Brooks D, et al. Cancer screening in the United States, 2016: A review of current American Cancer Society guidelines and current issues in cancer screening. CA Cancer J Clin 2016;66:96-114.

3. Soerjomataram I, Lortet-Tieulent J, Parkin DM, et al. Global burden of cancer in 2008: a systematic analysis of disability-adjusted life-years in 12 world regions. Lancet 2012;380:1840-50.

4. Ferlay J, Soerjomataram I, Dikshit R, et al. Cancer incidence and mortality worldwide: sources, methods and major patterns in GLOBOCAN 2012. Int J Cancer 2015;136:E359-86.

5. Sharma V, Karnes RJ. Prostatectomy versus Observation for Early Prostate Cancer. N Engl J Med 2017;377:1302.

6. Dimakakos A, Armakolas A, Koutsilieris M. Novel tools for prostate cancer prognosis, diagnosis, and follow-up. Biomed Res Int 2014;2014:890697.

7. Bibbins-Domingo K, Grossman DC, Curry SJ. The US Preventive Services Task Force 2017 Draft Recommendation Statement on Screening for Prostate Cancer: An Invitation to Review and Comment. JAMA 2017;317:1949-50.

8. Turkbey B, Brown AM, Sankineni S, et al. Multiparametric prostate magnetic resonance imaging in the evaluation of prostate cancer. CA Cancer J Clin 2016;66:326-36.

9. Ogino S, Lochhead P, Chan AT, et al. Molecular pathological epidemiology of epigenetics: emerging integrative science to analyze environment, host, and disease. Mod Pathol 2013;26:465-84.

10. Wan JCM, Massie C, Garcia-Corbacho J, et al. Liquid biopsies come of age: towards implementation of circulating tumour DNA. Nat Rev Cancer 2017;17:223-38.

11. Fukushige S, Horii A. DNA methylation in cancer: a gene silencing mechanism and the clinical potential of its biomarkers. Tohoku J Exp Med 2013;229:173-85.

12. Li S, Zhu Y, Ma C, et al. Downregulation of EphA5 by promoter methylation in human prostate cancer. BMC Cancer 2015;15:18.

13. Gevensleben H, Holmes EE, Goltz D, et al. PD-L1 promoter methylation is a prognostic biomarker for biochemical recurrence-free survival in prostate cancer patients following radical prostatectomy. Oncotarget 2016;7:79943-55.

14. Holmes EE, Goltz D, Sailer V, et al. PITX3 promoter methylation is a prognostic biomarker for biochemical recurrence-free survival in prostate cancer patients after radical prostatectomy. Clin Epigenetics 2016;8:104.

15. Lee J, Han JH, Jang A, et al. DNA Methylation-Mediated Downregulation of DEFB1 in Prostate Cancer Cells. PLoS One 2016;11:e0166664.

16. Gao X, Li LY, Rassler J, et al. Prospective Study of 
CRMP4 Promoter Methylation in Prostate Biopsies as a Predictor For Lymph Node Metastases. J Natl Cancer Inst 2017;109.

17. Uhl B, Gevensleben H, Tolkach Y, et al. PITX2 DNA Methylation as Biomarker for Individualized Risk Assessment of Prostate Cancer in Core Biopsies. J Mol Diagn 2017;19:107-14.

18. Cheyette BN, Waxman JS, Miller JR, et al. Dapper, a Dishevelled-associated antagonist of beta-catenin and JNK signaling, is required for notochord formation. Dev Cell 2002;2:449-61.

19. Fisher DA, Kivimäe S, Hoshino J, et al. Three Dact gene family members are expressed during embryonic development and in the adult brains of mice. Dev Dyn 2006;235:2620-30.

20. Yu Y, Yan W, Liu X, et al. DACT2 is frequently methylated in human gastric cancer and methylation of DACT2 activated Wnt signaling. Am J Cancer Res 2014;4:710-24.

21. Zhao Z, Herman JG, Brock MV, et al. Methylation of DACT2 promotes papillary thyroid cancer metastasis by activating Wnt signaling. PLoS One 2014;9:e112336.

22. Wang S, Dong Y, Zhang Y, et al. DACT2 is a functional tumor suppressor through inhibiting $W n t / \beta$-catenin pathway and associated with poor survival in colon cancer. Oncogene 2015;34:2575-85.

23. Zhang M, Linghu E, Zhan Q, et al. Methylation of

Cite this article as: Lan T, Yin L, Zhang H, Gu B, Ma P, Li $\mathrm{S}$, Li H. Diagnostic value of DACT-2 methylation in serum of prostate cancer patients. Ann Palliat Med 2021;10(3):2421-2428. doi: 10.21037/apm-20-1496
DACT2 accelerates esophageal cancer development by activating Wnt signaling. Oncotarget 2016;7:17957-69.

24. Li S, Yin L, Huang K, et al. Downregulation of DACT2 by Promoter Methylation and its Clinicopathological Significance in Prostate Cancer. J Cancer 2019;10:1755-63.

25. Shankar E, Bhaskaran N, MacLennan GT, et al. Inflammatory Signaling Involved in High-Fat Diet Induced Prostate Diseases. J Urol Res 2015;2:1018.

26. Diaz LA, Bardelli A. Liquid biopsies: genotyping circulating tumor DNA. J Clin Oncol 2014;32:579-86.

27. Alix-Panabières C, Pantel K. Clinical Applications of Circulating Tumor Cells and Circulating Tumor DNA as Liquid Biopsy. Cancer Discov 2016;6:479-91.

28. Leung F, Kulasingam V, Diamandis EP, et al. Circulating Tumor DNA as a Cancer Biomarker: Fact or Fiction. Clin Chem 2016;62:1054-60.

29. Shivapurkar N, Gazdar AF. DNA methylation based biomarkers in non-invasive cancer screening. Curr Mol Med 2010;10:123-32.

30. Warton K, Mahon KL, Samimi G. Methylated circulating tumor DNA in blood: power in cancer prognosis and response. Endocr Relat Cancer 2016;23:R157-71.

31. Xiang T, Fan Y, Li C, et al. DACT2 silencing by promoter CpG methylation disrupts its regulation of epithelial-tomesenchymal transition and cytoskeleton reorganization in breast cancer cells. Oncotarget 2016;7:70924-35. 